\title{
Exploring the surface properties of transneptunian objects and Centaurs with polarimetric FORS1/VLT observations ${ }^{\star}$
}

\author{
S. Bagnulo ${ }^{1}$, H. Boehnhardt ${ }^{2}$, K. Muinonen ${ }^{3}$, L. Kolokolova ${ }^{4}$, I. Belskaya ${ }^{5}$, and M. A. Barucci ${ }^{6}$ \\ 1 European Southern Observatory, Alonso de Cordova 3107, Vitacura, Santiago, Chile \\ e-mail: sbagnulo@eso.org \\ 2 Max-Planck-Institut für Sonnensystemforschung, Max-Planck-Strasse 2, 37191 Katlenburg-Lindau, Germany \\ e-mail: hboehnha@linmpi.mpg.de \\ 3 Observatory, PO Box 14, 00014 University of Helsinki, Finland \\ e-mail: muinonen@cc.helsinki.fi \\ 4 University of Maryland, College Park, MD, USA \\ e-mail: ludmilla@astro.umd.edu \\ 5 Astronomical observatory of Kharkiv National University, 35 Sumska Str., 61022 Kharkiv, Ukraine \\ e-mail: irina@astron.kharkov.ua \\ ${ }^{6}$ LESIA, Observatoire de Paris, 5 place Jules Janssen, 92195 Meudon Cedex, France \\ e-mail: antonella.barucci@obspm.fr
}

Received 15 November 2005 / Accepted 3 January 2006

\section{ABSTRACT}

Context. Polarization is a powerful remote-sensing method to investigate solar system bodies. It is an especially sensitive diagnostic tool to reveal physical properties of the bodies whose observational characteristics are governed by small scatterers (dust, regolith surfaces). For these objects, at small phase angles, a negative polarization is observed, i.e., the electric vector $\boldsymbol{E}$ oscillates predominantly in the scattering plane, contrary to what is typical for rather smooth homogeneous surfaces. The behavior of negative polarization with phase angle depends on the size, composition and packing of the scatterers. These characteristics can be unveiled by modelling the light scattering by the dust or regolith in terms of the coherent backscattering mechanism.

Aims. We investigate the surface properties of TNOs and Centaurs by means of polarimetric observations with FORS1 of the ESO VLT.

Methods. We have obtained new broadband polarimetric measurements over a range of phase angles for a TNO, 50000 Quaoar (in the $R$ Bessel filter), and a Centaur, 2060 Chiron (in the $B V R$ Bessel filters). Simultaneously to the polarimetry, we have obtained $R$ broadband photometry for both objects. We have modelled these new observations of Quaoar and Chiron, and revised the modelling of previous observations of the TNO 28978 Ixion using an improved value of its geometric albedo.

Results. TNOs Ixion and Quaoar, and Centaur Chiron show a negative polarization surge. The Centaur Chiron has the deepest polarization minimum (-1.5-1.4\%). The two TNOs show differing polarization curves: for Ixion, the negative polarization increases rapidly with phase; for Quaoar, the polarization is relatively small $(\simeq-0.6 \%)$, and nearly constant at the observed phase angles. For all three objects, modelling results suggest that the surface contains an areal mixture of at least two components with different single-scatterer albedos and photon mean-free paths.

Key words. Kuiper Belt - polarization

\section{Introduction}

Transneptunian objects (TNOs) in the Kuiper Belt are considered to represent one of the oldest and possibly most original population of solar system bodies that can be observed from Earth. Centaurs are escapees from the Kuiper Belt through gravitational interaction with Neptune and the other giant planets. They may eventually become members of the Jupiter family of comets, or may be ejected from the planet region due to

* Based on observations made with ESO Telescopes at the Paranal Observatory under programme ID 69.C-0133 and 073.C-0561 (PI: H. Boehnhardt). close encounters with the giant planets.

The intense study of physical properties of TNOs and Centaurs was triggered by the advent of large telescopes on the ground: besides a large set of photometric colours, also visible and near-IR spectra of a number of objects are available now. Polarimetric observations are more scarce: except for Pluto/Charon system (that was observed unresolved, e.g., by Kelsey \& Fix 1973), it was only recently that broadband polarized radiation of a TNO, the Plutino 28978 Ixion, has been observed and modelled (Boehnhardt et al. 2004).

Polarimetry is a powerful tool to investigate the physical properties of atmosphereless bodies. At small $\left(\leq 30^{\circ}\right)$ phase 
angles (the phase angle is the angle between the Sun and the observer as seen from the object), these objects exhibit a phenomenon of negative polarization: the observed flux perpendicular to the plane Sun-Object-Observer (the scattering plane) minus the observed flux perpendicular to that plane, divided by the sum of the two fluxes, turns to be a negative quantity. This phenomenon, first discovered through lunar observations by Lyot (1929), escapes from common sense interpretation, since elementary physics tells that reflected electric vector $\boldsymbol{E}$ oscillates predominantly in the plane perpendicular to the scattering plane rather than in the scattering plane. Solar-system objects show two types of angular dependence of negative polarization: either a smooth phase-angle change that has the minimum at $\sim 10^{\circ}$ (S-, C- asteroids, Moon) or a sharp surge with the minimum at $\sim 1-2^{\circ}$ (Saturn rings, Europa, E-asteroids) (see, e.g., Rosenbush et al. 2002). Both types of negative polarization, which also were observed in powdered laboratory samples, are currently interpreted in terms of enhanced backscattering of multiply scattered rays (Shkuratov 1989; Muinonen 1990).

Observations of negative polarization and simultaneous photometry of main-belt asteroids and other solar system bodies (see, e.g., Belskaya et al. 2005; Rosenbush et al. 2005) can be modelled to infer the properties of the surface texture of these objects. Faintness of the targets was the main obstacle hampering the same kind of study in TNOs and Centaurs ${ }^{1}$. Thanks to the advent of the large telescopes and instruments equipped with polarimetric capabilities, observations of TNOs and Centaurs are nowadays possible with signal to noise ratio comparable to that commonly reached for main-belt asteroid observations with small and middle-size telescopes.

After our first polarimetric study of 28978Ixion (Boehnhardt et al. 2004), in this paper we present new polarimetric and photometric measurements obtained with FORS1 at the ESO Very Large Telescope (VLT) for a TNO, 50000 Quaoar, and a Centaur, 2060 Chiron. We also present a revised modelling of the observed polarization and photometry of 28978 Ixion based on a determination of the geometric albedo that has been recently obtained, and that was not available at the time of our first modelling effort.

\section{Target summary}

Criteria used for target selection are that the targets are bright enough to allow us to measure the polarization with an error bar smaller than $0.05 \%$ in less than two hours telescope time. With FORS 1 at the ESO VLT, this sets the $R$ magnitude limit to about 20 . Another constraint is the possibility to observe the largest possible phase angle range. Complementary information on the geometric albedo and surface composition is essential for the modelling part. Moreover, it is desirable to study members of the various dynamical and taxonomic groups identified among the TNO population (Plutinos, Centaurs, classical and scattered-disk Objects). We finally selected three objects with known physical parameters (geometric albedos, colours,

\footnotetext{
1 Another difference in the observing and modelling techniques is that, due to the larger distance, the observed phase angle range is much smaller for TNOs and Centaurs than for main-belt asteroids
}

spectral slopes, and surface composition): Ixion, Quaoar, and Chiron.

\subsection{Ixion}

28978 Ixion, discovered in 2001, belongs to the dynamical class of Plutinos, and it is one of the largest known TNOs (400-550 km according to Stansberry et al. 2005). The visible spectrum by Marchi et al. (2003) is featureless with a gradient $S^{\prime}$ of $19.8 \% / 100 \mathrm{~nm}$. Optical and near-IR (Licandro et al. 2002) spectra have been interpreted by Boehnhardt et al. (2004) using an areal mixture of Titan tholin, amorphous carbon, water ice, and ice tholin. The same authors also present a surface model of Ixion based upon their $R$ filter polarimetry and simultaneous $R$ band photometry of the objects spanning the phase angle range $0.25^{\circ}-1.34^{\circ}$. In that work, an $R$-band geometric albedo of 0.1 was assumed for the modelling. Here we repeat the analysis for the higher $R$-band geometric albedo now available from Spitzer observations (0.23; Stansberry, priv. comm.). Further details about the properties of this object are given by Boehnhardt et al. (2004).

\subsection{Quaoar}

50000 Quaoar is a classical disk object in the Kuiper Belt. Orbital elements and red visible colours (Fornasier et al. 2004) suggest that the object could be a member of the "dynamically hot" population that is supposed to have migrated to the classical disk only after formation closer to the Sun (Gomes 2003). Apart from Pluto/Charon, 50000 Quaoar is the only TNO so far for that disk-resolved photometry could be performed: HST measurements allowed to determine the overall size and geometric albedo of the object to be $1260 \pm 190 \mathrm{~km}$, and about 0.1 , respectively (Brown \& Trujillo 2004). The photometric lightcurve of the object seems to be double-peaked with a period of about $17.6 \mathrm{~h}$ and an amplitude of $0.13 \mathrm{mag}$ suggesting an aspherical shape of the body and/or geometric albedo variations of the surface (Ortiz et al. 2003).

Quaoar visible spectra were obtained by Marchi et al. (2003) and by Fornasier et al. (2004). The reflectivity gradients $S^{\prime}$ obtained in the two papers are not fully consistent, and their mean value is $27.6 \pm 0.3 \% / 100 \mathrm{~nm}$. Visible spectrum appears to be featureless.

Quaoar has been observed also in the near-infrared by Jewitt \& Luu (2004) at the Subaru $8 \mathrm{~m}$ telescope. The complete spectrum shows a positive-slope continuum from 0.4 up to $1.3 \mu \mathrm{m}$, that is considered typical for the presence of organic materials on its surface. The spectrum shows strong absorption bands at 1.5 and $2.0 \mu \mathrm{m}$ due to $\mathrm{H}_{2} \mathrm{O}$ ice with the band at $1.65 \mu \mathrm{m}$ typical for the crystalline structure in the ice. A small presence of ammonia hydrate has also been supposed on the basis of the presence of faint features at $2.2 \mu \mathrm{m}$ (detected also by Pinilla-Alonso et al. 2004). This was the first time that the quality of a TNO spectrum was good enough to distinguish between crystalline and amorphous ice. The detection of crystalline ice indicates that the temperature has reached at least $110 \mathrm{~K}$ (critical temperature necessary for crystallization). This object is 
large enough to be cryovolcanically active, and crystalline ice and ammonia hydrate might be products of this type of activity. Jewitt \& Luu (2004) suggested that Quaoar has been recently resurfaced either by impacts or by cryovolcanic outgassing or by a combination of these two processes.

\subsection{Chiron}

2060 Chiron is the first discovered (1977) and best observed Centaur. The intermediate character of the object between TNOs and comets is apparent from photometric observations that show recurrent episodes of coma activity (gas and dust) and of stellar appearance (see for example Meech \& Belton 1990; Luu \& Jewitt 1990; Bus et al. 1991; Duffard et al. 2002).

Chiron was observed spectroscopically in the visible region by many authors (see Barucci et al. 2003) showing a flat spectrum with no absorption features. The reflectivity gradient $S^{\prime}$, which ranges between -0.2 up to $2.3 \pm 0.1 \% / 100 \mathrm{~nm}$, seems more similar to that of C-type asteroids rather than to the mean reflectance slope of cometary nuclei. The small variation on the optical reflectivity gradient could be due to dust production variation connected to episodes of recurrent cometary activity.

Several spectra obtained in the near-infrared did not show any features. Only Foster et al. (1999) and Luu et al. (2000) detected a $2 \mu \mathrm{m}$ absorption band suggesting the presence of $\mathrm{H}_{2} \mathrm{O}$ ice on Chiron's surface. Later on, Romon-Martin et al. (2003) observed Chiron again during high activity in the visible and NIR showing a flat behaviour without any spectral features finding that is compatible with the hypothesis made by Luu et al. (2000) that the detection of water ice in Chiron spectra would be correlated with its cometary activity level. Such activity could cause a rain of cometary debris on its surface changing the surface mantle. The ice present on the surface, is probably mixed with dark impurities which mask the spectral bands.

Nucleus properties using multi-wavelength information has revealed an about $70 \mathrm{~km}$ nucleus of relatively bright geometric albedo (0.17) and moderate axis ratio (1.16) or surface albedo variations (Groussin et al. 2004).

From the large number of publications on Chiron (more than 150 to-date) several interesting properties of 2060 Chiron have been worked out. However, a synoptic picture of the nucleus and its surface properties has not yet evolved.

\section{New observations with FORS1}

Observations of Chiron and Quaoar have been obtained at the ESO VLT with the FORS1 instrument in service mode during the observing period from April to September 2004. Until June 1, 2004, FORS1 was attached at the VLT Unit Telescope 1 (Antu). After that date, FORS1 was moved to the VLT Unit Telescope 2 (Kueyen).

FORS1 is a multi-mode instrument for imaging and (multiobject) spectroscopy equipped with polarimetric optics. For the present study, FORS1 has been used to measure the broadband polarization of Chiron at six different epochs in the Bessel
$B V R$ filters, and to measure the broadband polarization of Quaoar at five different epochs in the Bessel $R$ filter (Sect. 3.1). In fact, one series of Chiron measurements was started on night 2004-08-05/06 and aborted after the observations in the $R$ filter because the seeing conditions were too good $\left(\$ 0.6^{\prime \prime}\right)$ with consequent risk of CCD saturation. The series was repeated the following night. From target acquisition images, a by-product of polarimetric observations, we could also obtain photometry in the Bessel $R$ filter (Sect. 3.2). Differential tracking was used for all our observations, so that long exposure time images of Chiron, obtained in polarimetric mode, could be combined altogether to search for coma activity (Sect. 3.3).

Taking advantage of the flexibility offered by the "VLT service observing mode", we distributed the observations along a few months as to obtain data points approximately equally spread over the phase angle ranges of the targets. We set precise time intervals for the execution of the observations. In presence of the Moon, the sky-background is highly polarized, hence we generally tried to avoid observations with the target close to the Moon, and with a too large fraction of lunar illumination. However, a posteriori we found that the presence of the Moon did not jeopardize our observations. The log of the observations can be inferred from Tables 1 to 4 .

\subsection{Polarimetry}

To perform linear polarization measurements, a $\lambda / 2$ retarder waveplate and a Wollaston prism are inserted in the FORS1 optical path (see Appenzeller 1967). The $\lambda / 2$ retarder waveplate can be rotated in $22.5^{\circ}$ steps. Stokes $Q$ and $U$ parameters (defined as in Shurcliff 1962) are measured by combining the photon counts (background subtracted) of ordinary and extra-ordinary beams ( $f^{\mathrm{o}}$ and $f^{\mathrm{e}}$, respectively) observed at various retarder waveplate positions $\alpha$, where $\alpha$ indicates the angle between the acceptance axis of the ordinary beam of the Wollaston prism and the fast axis of the retarder waveplate.

In the following, we will always work with the ratios $Q / I$ and $U / I$, and will adopt the notation:

$P_{Q}=\frac{Q}{I}$ and $P_{U}=\frac{U}{I}$

In the ideal case, $P_{Q}$ is obtained measuring the quantity

$r=(-1)^{k} \frac{f^{\mathrm{o}}-f^{\mathrm{e}}}{f^{\mathrm{o}}+f^{\mathrm{e}}}$

at any retarder waveplate position $\alpha=k 45^{\circ}$, and $P_{U}$ is obtained measuring the ratio $r$ at any position $\alpha=k 45^{\circ}+22.5^{\circ}$ $(k=0,1,2, \ldots, 7)$. The validity of this assertion can be verified e.g. with the help of Eq. (1.33) of Landi Degl'Innocenti \& Landolfi (2004). In practice, there are several deviations from the ideal case. For instance, the actual retardance value of the retarder waveplate may deviate from the nominal $\pi$ value; the transmission of the ordinary and extraordinary beam are not identical, even after flat fielding correction. The effect of these 
(and other) sources of instrumental polarization can be largely reduced at the first order by measuring

$$
\begin{aligned}
& P_{Q}^{i j}=\frac{1}{2}\left\{\left(\frac{f^{\mathrm{o}}-f^{\mathrm{e}}}{f^{\mathrm{o}}+f^{\mathrm{e}}}\right)_{\alpha=2(i-1) \times 45^{\circ}}-\left(\frac{f^{\mathrm{o}}-f^{\mathrm{e}}}{f^{\mathrm{o}}+f^{\mathrm{e}}}\right)_{\alpha=(2 j-1) \times 45^{\circ}}\right\} \\
& P_{U}^{i j}=\frac{1}{2}\left\{\left(\frac{f^{\mathrm{o}}-f^{\mathrm{e}}}{f^{\mathrm{o}}+f^{\mathrm{e}}}\right)_{\alpha=2(i-1) \times 45^{\circ}+22.5^{\circ}}-\left(\frac{f^{\mathrm{o}}-f^{\mathrm{e}}}{f^{\mathrm{o}}+f^{\mathrm{e}}}\right)_{\alpha=(2 j-1) \times 45^{\circ}+22.5^{\circ}}\right\}
\end{aligned}
$$

where $i$ and $j$ are integers numbers ${ }^{2}$. In the simplest case, linear polarization can be measured from the observations obtained at four angles of the retarder waveplate:

$$
\begin{aligned}
& P_{Q}=\frac{1}{2}\left\{\left(\frac{f^{\circ}-f^{\mathrm{e}}}{f^{\mathrm{o}}+f^{\mathrm{e}}}\right)_{\alpha=0^{\circ}}-\left(\frac{f^{\mathrm{o}}-f^{\mathrm{e}}}{f^{\mathrm{o}}+f^{\mathrm{e}}}\right)_{\alpha=45^{\circ}}\right\} \\
& P_{U}=\frac{1}{2}\left\{\left(\frac{f^{\mathrm{o}}-f^{\mathrm{e}}}{f^{\mathrm{o}}+f^{\mathrm{e}}}\right)_{\alpha=22.5^{\circ}}-\left(\frac{f^{\mathrm{o}}-f^{\mathrm{e}}}{f^{\mathrm{o}}+f^{\mathrm{e}}}\right)_{\left.\alpha=67.5^{\circ}\right\} .}\right.
\end{aligned}
$$

It is convenient (and recommended in the FORS1/2 user manual) to obtain Stokes $Q$ and $U$ adding up observations obtained with the retarder waveplate at various positions:

$$
\begin{aligned}
& P_{Q}=\frac{1}{m} \sum_{l=1}^{m} P_{Q}^{l l} \\
& P_{U}=\frac{1}{m} \sum_{l=1}^{m} P_{U}^{l l},
\end{aligned}
$$

where $m$ represents the number of pairs of observations for each Stokes parameter, and $P_{Q}^{l l}$ and $P_{U}^{l l}$ are obtained from Eq. (1) setting $i=j=l$. We performed simple numerical simulations to study the impact on the precision of the polarimetric measurements of a deviation of the waveplate retardance from its nominal value $\left(180^{\circ}\right)$. We found that using Eq. (2) with $m=2$, a deviation from the nominal value of the waveplate retardance as large as $5^{\circ}$, for the polarization value observed in our targets, would introduce a spurious contribution $\ll 0.01 \%$. Figure 4.1 of FORS1/2 user manual shows that actual deviation of the retarder waveplate from the nominal value is well within $5^{\circ}$. We conclude that in our data, the effect of instrumental polarization due to the chromathism of the retarder waveplate is negligible.

It should be noted that FORS1 is affected by a problem with spurious linear instrumental polarization that cannot be eliminated even by using the reduction technique explained above. This spurious polarization, due to the presence of rather curved lenses in the collimator, combined with the non complete removal of reflections by the coatings, is axially symmetric, and smoothly increases from less than $0.03 \%$ on the optical axis to $0.7 \%$ at an axis distance of $3 \operatorname{arcmin}$ (in the $V$ band). This problem has been discovered and investigated by Patat $\&$ Romaniello (2006). In our case, since our targets are always in the center of the field of view, the problem of the instrumental polarization can be safely ignored.

The error-bar on the $P_{Q}$ or $P_{U}$ measured from a pair of observations is

$$
\begin{aligned}
\sigma_{X^{i j}}^{2}= & \left(\left(\frac{f^{\mathrm{e}}}{\left(f^{\mathrm{o}}+f^{\mathrm{e}}\right)^{2}}\right)^{2} \sigma_{f^{\circ}}^{2}+\left(\frac{f^{\circ}}{\left(f^{\circ}+f^{\mathrm{e}}\right)^{2}}\right)^{2} \sigma_{f^{\mathrm{e}}}^{2}\right)_{\alpha=2(i-1) \times 45^{\circ}+\phi_{0}} \\
& +\left(\left(\frac{f^{\mathrm{e}}}{\left(f^{\mathrm{o}}+f^{\mathrm{e}}\right)^{2}}\right)^{2} \sigma_{f^{\circ}}^{2}+\left(\frac{f^{\mathrm{o}}}{\left(f^{\circ}+f^{\mathrm{e}}\right)^{2}}\right)^{2} \sigma_{f^{\mathrm{e}}}^{2}\right)_{\alpha=(2 j-1) \times 45^{\circ}+\phi_{0}},
\end{aligned}
$$

${ }^{2}$ We note that in a similar formula that we reported in Boehnhardt et al. (2004), the factor 2 in the indices that denote the angles is missing because of a typo. where $\phi_{0}=0$ in case $X$ represents $P_{Q}$ and $\phi_{0}=22.5^{\circ}$ in case $X$ represents $P_{U}$. When $P_{Q}$ and $P_{U}$ are obtained adding up $m$ pairs as in Eq. (2), the error is given by

$\sigma_{X}^{2}=\frac{1}{m} \sum_{l=1}^{m} \sigma_{X^{l l}}^{2}$.

For the sake of simplicity, assuming $f^{\mathrm{o}}=f^{\mathrm{e}}=\mathcal{N}$ and also assuming $\mathcal{N}$ to be the same for all positions of the retarder waveplate, we obtain, neglecting the errors due to sky-subtraction

$\sigma_{X}=\frac{1}{2} \frac{1}{\sqrt{m \mathcal{N}}}$

where $\sqrt{\mathcal{N}}$ represents the signal to noise ratio (SNR) measured in the individual beam for each of the $m$ exposures (in other words, $\sqrt{m \mathcal{N}}$ is the cumulative SNR in each beam). It appears for instance that to get an error bar on the Stokes parameter $P_{Q}$ or $P_{U}$ of about $0.1 \%$, one should take a pair of exposures with SNR of about 500 each (integrated over the individual pointspread function area of each beam of each exposure).

When multiple pairs of exposures are taken, it is useful to study the distribution of the $P_{Q}^{i j}\left(P_{U}^{i j}\right)$ values obtained substituting $i, j=1,2, \ldots m$. In particular, a $\sigma$-clipping algorithm can be applied to the $P_{Q}^{i j}$ and $P_{U}^{i j}$ distributions in order to "clean" the data, by rejecting those values that deviate more than a certain distance from the median (see Boehnhardt et al. 2004; Bagnulo et al. 2006).

Stokes $Q$ and $U$ are usually measured with the instrument position angle $=0^{\circ}$, i.e. to have the acceptance axis of the ordinary beam of the Wollaston prism aligned to the North Celestial Meridian (and the acceptance axis of the extra-ordinary beam perpendicular to it). We then transform the Stokes parameters according to

$$
\begin{aligned}
& P_{Q}^{\prime}=\cos (2(\Phi+\pi / 2)) P_{Q}+\sin (2(\Phi+\pi / 2)) P_{U} \\
& P_{U}^{\prime}=-\sin (2(\Phi+\pi / 2)) P_{Q}+\cos (2(\Phi+\pi / 2)) P_{U}
\end{aligned}
$$

where $\Phi$ is the angle between the direction Object-North Pole and the direction Object-Sun. This angle can be calculated applying the four parts formula to the spherical triangle defined by the object (with coordinates $\alpha_{\mathrm{T}}, \delta_{\mathrm{T}}$ ), the Sun (with coordinates $\alpha_{\odot}, \delta_{\odot}$ ) and the North celestial pole:

$\sin \delta_{\mathrm{T}} \cos \left(\alpha_{\odot}-\alpha_{\mathrm{T}}\right)=\cos \left(\delta_{\mathrm{T}}\right) \tan \left(\delta_{\odot}\right)-\sin \left(\alpha_{\odot}-\alpha_{\mathrm{T}}\right) \frac{1}{\tan (\Phi)}$.

This way $P_{Q}^{\prime}$ represents the flux perpendicular to the plane SunObject-Earth (the scattering plane) minus the flux parallel to that plane, divided by the sum of the two fluxes. The angle of maximum polarization is obtained as

$\Theta^{\prime}=\frac{1}{2} \arctan \left(\frac{P_{U}^{\prime}}{P_{Q}^{\prime}}\right)+\Theta_{0}^{\prime}$

where

$\Theta_{0}^{\prime}= \begin{cases}0 & \text { if } P_{Q}^{\prime}>0 \text { and } P_{U}^{\prime} \geq 0 \\ \pi & \text { if } P_{Q}^{\prime}>0 \text { and } P_{U}^{\prime}<0 . \\ \pi / 2 & \text { if } P_{Q}^{\prime}<0\end{cases}$

or

$\Theta^{\prime}= \begin{cases}\pi / 4 & \text { if } P_{Q}^{\prime}=0 \text { and } P_{U}^{\prime}>0 \\ 3 \pi / 4 & \text { if } P_{Q}^{\prime}=0 \text { and } P_{U}^{\prime}<0\end{cases}$ 
(see Landi Degl'Innocenti \& Landolfi 2004.) Incidentally, it should be noted that the $\Theta_{0}^{\prime}$ term is occasionally incorrectly neglected.

Observations of Chiron were performed with the retarder waveplate at all positions between 0 and $157.5^{\circ}$ (at $22.5^{\circ}$ steps), i.e., setting $m=2$ in Eq. (2), using the broadband Bessel $B, V$, and $R$ filters. For Quaoar we used all positions of the retarder waveplate from $0^{\circ}$ to $337.5^{\circ}$, i.e., setting $m=4$ in Eq. (2), using the Bessel $R$ filter. For each frame, exposure times $t$ were as follows. For Chiron we set $t=460,140$, and $110 \mathrm{~s}$ in the $B$, $V$, and $R$ filter, respectively. For Quaoar ( $R$ filter only), we set $t=250 \mathrm{~s}$. Note that, for each Stokes parameter we obtained 2 pairs of exposures for Chiron, and 4 pairs of exposures for Quaoar. Therefore the total shutter time for Chiron observations was of about 60,20 , and $15 \mathrm{~min}$ in the $B, V$, and $R$ filter, respectively, and of about $67 \mathrm{~min}$ for Quaoar ( $R$ filter only). For each observation of Chiron, the typical SNR accumulated in each beam was about 1700,1600 , and 1600 , in the $B, V$, and $R$ filters respectively. For each observation of Quaoar, in the $R$ filter, we obtained a SNR accumulated in each beam of about 1400 .

The photon counts $f^{\mathrm{o}}$ and $f^{\mathrm{e}}$ were measured via simple aperture photometry performed on the images, obtained after bias subtraction and flatfield correction (master flat field was obtained from sky images obtained during twilight with no polarimetric optics in). More sophisticated methods based on point spread function (PSF) fitting are difficult to apply because of the star trailing due to differential tracking on the moving targets. Sky background was measured in an annulus with $5^{\prime \prime}$ inner radius, centered around the source, of $2^{\prime \prime}$ to 6 " width. The errors on $f^{\circ}$ and $f^{\mathrm{e}}$ were estimated as explained in the Sect. 3.3.5.8 of Davis (1987). $f^{\mathrm{o}}, f^{\mathrm{e}}$, and their error estimates were measured for aperture values ranging from $0.6^{\prime \prime}$ to $4^{\prime \prime}$.

$P_{Q}$ and $P_{U}$ values were found to be slightly dependent on the aperture adopted to measure $f^{\mathrm{o}}$ and $f^{\mathrm{e}}$. This effect was more critical when the target was in a crowded field and/or in presence of strong background polarization by the Moon. The final value was selected as the one for which the error on $P_{Q}$ and $P_{U}$ was minimum, i.e., usually for aperture values between $1.2^{\prime \prime}$ and $2.5^{\prime \prime}$.

The results of the polarimetric measurements are given in Tables 1 and 2, that are organized as follows. Columns 1 and 2 give the epoch of the observations (date and UT time).

Columns 3 and 4 give Moon angular distance and fraction of lunar illumination (FLI), respectively.

Column 5 gives an estimate of the night time sky conditions: $\mathrm{THN}=$ thin cirrus, $\mathrm{CLR}=$ clear, $\mathrm{PHO}=$ photometric. The classification given in these tables is based on our inspection of reduction products and of the atmospheric monitors of the observatory at http://www . eso.org/ gen-fac/pubs/astclim/forecast/meteo/CIRA/images/ repository/lossam/ and is somewhat arbitrary. Those nights when no photometric standard stars were observed, or when the zeropoints were not considered stable for the entire night, are indicated with a question mark. Sky transparency does not affect the precision of the polarization measurements, but it does affect the precision of the photometry (see Tables 3 and 4). Note that the precision of the measurements (both polarimetric and photometric) depends also on how the field of view close to the target is crowded with background objects. This situation of course changes from epoch to epoch. Observations labelled with (c) are hampered to some extent by a crowded background. In the case of Chiron observations on 2004-09-01 the situation was probably especially critical.

Column 6 gives the Sun-Target-Observer angle, i.e., the target's apparent phase angle as seen at observer's location, expressed in degrees. This phase angle was obtained from the object ephemeris calculated at the JPL's solar system dynamics WWW site at http://ssd.jpl.nasa.gov/.

Column 7 specifies the broadband filter used for the observations.

Columns 8-10 give $P_{Q}^{\prime}, P_{U}^{\prime}$, and the angle of maximum polarization $\Theta^{\prime}$, respectively, after the transformation of Eq. (5). It should be noted that for both Chiron and Quaoar, the measured $P_{U}^{\prime}$ is generally consistent with zero (equivalent to the fact that $\Theta^{\prime}$ is generally consistent with $90^{\circ}$ ). This means that the principal axes of the polarization ellipse are aligned with the coordinate axes perpendicular and parallel to the scattering plane. Furthermore, the observed $P_{Q}^{\prime}$, i.e., the flux perpendicular to the scattering plane minus the flux parallel to that plane, divided by the sum of the two fluxes, is always a negative quantity. This means that the direction of the polarization is included in the scattering plane (this case is normally referred to as "negative polarization"), in contrast to what is expected for a dielectric medium, i.e., that the direction of polarization be perpendicular to the scattering plane (this case is normally referred to as "positive polarization").

Polarimetric measurements of Quaoar and Chiron are plotted against object phase angle in Figs. 3 and 4. As far as Chiron observations are concerned, it appears that the observed polarization does not depend on the filter used, at least when considering the error bars of the observations. The absolute value of the polarization increases as the phase angle decreases, perhaps reaching a minimum around phase $1.5-2.0 \mathrm{deg}$. Observations of this object at smaller phase angles were unfortunately not taken although originally scheduled in our service observing campaign.

\subsection{Photometry}

The object magnitudes were measured in the acquisition images obtained in the $R$ Bessel filter with no polarimetric optics in the light path. Exposure times were $5 \mathrm{~s}$ for Chiron, and $30 \mathrm{~s}$ for Quaoar.

Since our program was aimed at polarization measurements, we did not obtain a number of observations of photometric standard stars sufficient to estimate precise zeropoints and extinction coefficients for the various nights. In most of the cases, the zeropoints were obtained from only one frame obtained during the night when our observations were executed (and not necessarily close to them in time). In some cases, no photometric standard stars were observed at all (see Tables 1 and 2). In these cases we adopted the values measured in the nearest night with similar sky conditions. For the extinction coefficient $K_{R}$ and the colour term $k_{V R}$ we used the 
Table 1. The observed polarization of 2060 Chiron. The meaning of the various columns is given in the text.

\begin{tabular}{|c|c|c|c|c|c|c|c|c|c|}
\hline $\begin{array}{c}\text { Date } \\
\text { (yyyy } \mathrm{mm} \mathrm{dd} \text { ) }\end{array}$ & $\begin{array}{c}\text { Time (UT) } \\
\text { (hh:mm) }\end{array}$ & $\begin{array}{l}\text { Moon } \\
\text { dist. }\end{array}$ & FLI & $\begin{array}{l}\text { Sky } \\
\text { transp. }\end{array}$ & $\begin{array}{l}\text { Phase angle } \\
\text { (DEG) }\end{array}$ & Filter & $\begin{array}{l}P_{Q}^{\prime} \\
(\%)\end{array}$ & $\begin{array}{l}P_{U}^{\prime} \\
(\%)\end{array}$ & $\begin{array}{c}\Theta^{\prime} \\
(\mathrm{DEG})\end{array}$ \\
\hline 20040527 & $06: 37$ & $139^{\circ}$ & 0.3 & CLR & 3.469 & $\bar{R}$ & $-1.14 \pm 0.04$ & $0.02 \pm 0.04$ & $89.4 \pm 1.0$ \\
\hline 20040527 & 07:03 & & & & 3.468 & V & $-1.20 \pm 0.04$ & $0.13 \pm 0.04$ & $86.9 \pm 0.9$ \\
\hline 20040527 & $07: 52$ & & & & 3.467 & $B$ & $-1.24 \pm 0.04$ & $-0.04 \pm 0.05$ & $90.9 \pm 1.1$ \\
\hline 20040629 & 03:19 & $64^{\circ}$ & 0.4 & CLR? & 1.410 & $R$ & $-1.40 \pm 0.05$ & $0.05 \pm 0.04$ & $89.1 \pm 0.8$ \\
\hline 20040629 & 03:44 & & & & 1.409 & V & $-1.35 \pm 0.05$ & $-0.08 \pm 0.04$ & $91.6 \pm 0.9$ \\
\hline 20040629 & $04: 33$ & & & & 1.407 & $B$ & $-1.32 \pm 0.04$ & $0.08 \pm 0.05$ & $88.2 \pm 1.2$ \\
\hline 20040806 & 05:53 & $94^{\circ}$ & 0.7 & PHO & 1.766 & $R$ & $-1.40 \pm 0.04$ & $0.00 \pm 0.04$ & $89.9 \pm 0.8$ \\
\hline 20040807 & $03: 46$ & $105^{\circ}$ & 0.7 & THN (c) & 1.828 & $R$ & $-1.33 \pm 0.04$ & $-0.01 \pm 0.04$ & $90.2 \pm 0.8$ \\
\hline 20040807 & $04: 13$ & & & & 1.829 & V & $-1.46 \pm 0.03$ & $-0.14 \pm 0.03$ & $92.7 \pm 0.7$ \\
\hline 20040807 & 05:00 & & & & 1.832 & $B$ & $-1.52 \pm 0.04$ & $-0.09 \pm 0.05$ & $91.8 \pm 0.9$ \\
\hline 20040813 & $00: 17$ & $167^{\circ}$ & 0.9 & PHO (c) & 2.219 & $R$ & $-1.33 \pm 0.04$ & $0.08 \pm 0.04$ & $88.3 \pm 1.0$ \\
\hline 20040813 & $00: 42$ & & & & 2.221 & V & $-1.34 \pm 0.04$ & $-0.08 \pm 0.04$ & $91.6 \pm 0.9$ \\
\hline 20040813 & 01:31 & & & & 2.223 & $B$ & $-1.45 \pm 0.03$ & $-0.19 \pm 0.05$ & $93.8 \pm 1.1$ \\
\hline 20040901 & $00: 56$ & $74^{\circ}$ & 0.5 & THN? (c) & 3.327 & $R$ & $-1.12 \pm 0.04$ & $0.05 \pm 0.04$ & $88.7 \pm 1.1$ \\
\hline 20040901 & $01: 21$ & & & & 3.328 & V & $-1.09 \pm 0.05$ & $-0.08 \pm 0.04$ & $92.1 \pm 1.2$ \\
\hline 20040901 & 02:10 & & & & 3.330 & $B$ & $-1.15 \pm 0.05$ & $-0.24 \pm 0.06$ & $95.9 \pm 1.6$ \\
\hline 20040927 & $01: 24$ & $56^{\circ}$ & 0.4 & CLR? & 4.232 & $R$ & $-0.89 \pm 0.07$ & $0.03 \pm 0.05$ & $89.1 \pm 1.8$ \\
\hline 20040927 & 01:49 & & & & 4.232 & V & $-1.05 \pm 0.05$ & $-0.20 \pm 0.05$ & $95.4 \pm 1.7$ \\
\hline 20040927 & $02: 38$ & & & & 4.233 & $B$ & $-0.87 \pm 0.09$ & $-0.10 \pm 0.08$ & $93.3 \pm 3.0$ \\
\hline
\end{tabular}

Table 2. The observed polarization of 50000 Quaoar. The meaning of the various columns is given in the text.

\begin{tabular}{cccclcccrr}
\hline \hline $\begin{array}{c}\text { Date } \\
\text { (yyyy mm dd) }\end{array}$ & $\begin{array}{c}\text { Time (UT) } \\
\text { (hh:mm) }\end{array}$ & $\begin{array}{c}\text { Moon } \\
\text { dist. }\end{array}$ & FLI & $\begin{array}{l}\text { Sky } \\
\text { transp. }\end{array}$ & $\begin{array}{c}\text { Phase angle } \\
\text { (DEG) }\end{array}$ & Filter & \multicolumn{1}{c}{$\begin{array}{c}P_{Q}^{\prime} \\
(\%)\end{array}$} & \multicolumn{1}{c}{$\begin{array}{c}P_{U}^{\prime} \\
(\%)\end{array}$} & $\begin{array}{r}\Theta^{\prime} \\
(\mathrm{DEG})\end{array}$ \\
\hline 20040418 & $04: 43$ & $47^{\circ}$ & 1.0 & CLR & 0.952 & $R$ & $-0.64 \pm 0.05$ & $0.14 \pm 0.08$ & $83.9 \pm 3.9$ \\
20040513 & $07: 38$ & $93^{\circ}$ & 0.8 & CLR? & 0.496 & $R$ & $-0.50 \pm 0.06$ & $-0.14 \pm 0.05$ & $97.7 \pm 3.6$ \\
20040526 & $03: 37$ & $107^{\circ}$ & 0.2 & PHO & 0.252 & $R$ & $-0.49 \pm 0.06$ & $0.10 \pm 0.05$ & $84.3 \pm 3.5$ \\
20040710 & $01: 44$ & $118^{\circ}$ & 0.7 & PHO (c) & 0.797 & $R$ & $-0.53 \pm 0.04$ & $0.09 \pm 0.05$ & $85.3 \pm 3.1$ \\
20040811 & $02: 34$ & $75^{\circ}$ & 0.8 & CLR? & 1.231 & $R$ & $-0.65 \pm 0.04$ & $-0.01 \pm 0.06$ & $90.4 \pm 2.6$ \\
\hline
\end{tabular}

values estimated for the P73 ESO observing period published at http://www . eso.org/observing/dfo/quality/FORS1/ qc/photcoeff/photcoeffs_fors1.html i.e.,

$K_{R}=0.045 \pm 0.019$

$k_{V R}=0.0090 \pm 0.0017$.

For the Quaoar colour indices we adopted $V-R=0.64 \pm 0.01$ (Tegler et al. 2003), and for Chiron $V-R=0.37 \pm 0.03$ (Davies et al. 1998). Finally, reduced magnitude $R$ of the target was obtained by taking into account the distance Sun-Object $r$ and the distance Earth-Object $\Delta$ at the epoch of the observations, and calculating the reduced magnitude

$$
R=m_{R}-5 \log (r)-5 \log (\Delta)
$$

where $m_{R}$ is the observed $R$-band magnitude.

The results of our photometry are given in Tables 3 and 4 .

From a linear least-square fit of the data, we found that for Chiron the $H_{R}$ absolute magnitude at zero phase angle is $5.52 \pm 0.07 \mathrm{mag}$, and the slope of the opposition surge is $0.045 \pm 0.023 \mathrm{mag} / \mathrm{deg}$. Previous numerous photometric observations of Chiron (for summary see Groussin et al. 2004) have shown considerable variations of absolute magnitude with heliocentric distance attributed to its sporadic cometary behavior. The absolute magnitude of Chiron in 2004 is brighter as compared to the observations in 1988-1990 made at the same heliocentric distances (Bus et al. 1991). It may indicate that we observed Chiron closely after an activity period which may have caused partial resurfacing of the object. This is why we paid a special attention to search for a coma around Chiron (see Sect. 3.3).

For Quaoar, the $H_{R}$ absolute magnitude at zero phase angle, obtained from a linear fit is $2.16 \pm 0.05 \mathrm{mag}$, and the linear slope is $0.16 \pm 0.06 \mathrm{mag} / \mathrm{deg}$. The determined value of absolute magnitude coincides with that used for Quaoar for geometric albedo determination (Brown \& Trujillo 2004).

Note that $H_{R}$ absolute magnitudes at zero phase angles and linear slopes will be re-estimated based on our modelling technique in Sect. 4.

\subsection{Search for a coma around 2060 Chiron}

Visual inspection and measurements of the full-width-at-halfmaximum of the Chiron and neighbouring star images did not 
Table 3. The observed $R$ photometry of 2060 Chiron.

\begin{tabular}{ccccc}
\hline \hline $\begin{array}{c}\text { Date } \\
\text { (yyy mm dd) }\end{array}$ & $\begin{array}{c}\text { Time (UT) } \\
(\mathrm{hh}: \mathrm{mm})\end{array}$ & $\begin{array}{c}\text { Phase angle } \\
(\mathrm{DEG})\end{array}$ & $\begin{array}{c}m_{R} \\
(\mathrm{mag})\end{array}$ & $\begin{array}{c}R \\
(\mathrm{mag})\end{array}$ \\
\hline 20040527 & $06: 23$ & 3.470 & $16.64 \pm 0.04$ & 5.68 \\
20040629 & $03: 04$ & 1.411 & $16.48 \pm 0.05$ & 5.56 \\
20040806 & $05: 40$ & 1.766 & $16.49 \pm 0.04$ & 5.54 \\
20040807 & $03: 33$ & 1.828 & $16.65 \pm 0.04$ & 5.69 \\
20040813 & $00: 02$ & 2.219 & $16.55 \pm 0.05$ & 5.58 \\
20040901 & $00: 39$ & 3.326 & $16.65 \pm 0.04$ & 5.63 \\
20040927 & $01: 10$ & 4.232 & $16.84 \pm 0.04$ & 5.74 \\
\hline
\end{tabular}

Table 4. The observed $R$ photometry of 50000 Quaoar.

\begin{tabular}{ccccc}
\hline \hline $\begin{array}{c}\text { Date } \\
\text { (yyyy mm dd) }\end{array}$ & $\begin{array}{c}\text { Time (UT) } \\
(\text { hh:mm) }\end{array}$ & $\begin{array}{c}\text { Phase angle } \\
(\mathrm{DEG})\end{array}$ & $\begin{array}{c}m_{R} \\
(\mathrm{mag})\end{array}$ & $\begin{array}{c}R \\
(\mathrm{mag})\end{array}$ \\
\hline 20040418 & $03: 59$ & 0.952 & $18.60 \pm 0.05$ & 2.27 \\
20040513 & $06: 54$ & 0.497 & $18.60 \pm 0.04$ & 2.27 \\
20040526 & $02: 52$ & 0.252 & $18.51 \pm 0.04$ & 2.19 \\
20040710 & $00: 59$ & 0.796 & $18.57 \pm 0.04$ & 2.24 \\
20040811 & $01: 49$ & 1.231 & $18.73 \pm 0.04$ & 2.38 \\
\hline
\end{tabular}

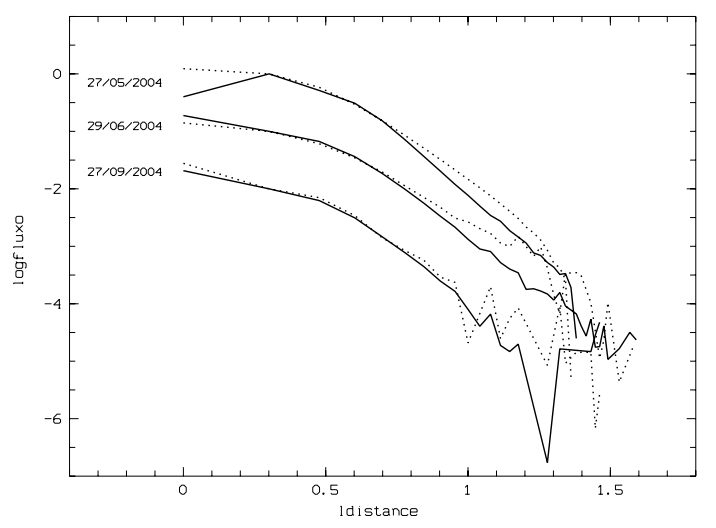

Fig. 1. Coma search around 2060 Chiron. Solid lines show the Chiron radial profiles, the dotted lines show the radial profiles of the comparison stars. The $x$-axis represents the logarithmic distance in pixels from the centre of the Chiron (or the stars) PSF images. The $y$-axis represents the flux (in arbitrary units).

reveal any indications for the presence of a coma around the Centaur. For a more thorough analysis we calculated the radial flux profile measured in concentric rings around the object, and compared it with the radial profile across the star trails, averaged along the trail direction and for both sides of the trail. The resulting object and star profiles are normalized to unity brightness at "one pixel" central distance and to zero at background distance 30 pixels from the center. Figure 1 shows the results for three observing dates (for other dates the presence of star trails close to the object image jeopardized the accuracy of this analysis). Since the radial profile of Chiron (solid line) is basically identical to that of the comparison stars (dotted line) or falls at slightly lower flux levels, we conclude that a coma around Chiron is not present or it is well beyond our detection limit (order of $29 \mathrm{mag} / \operatorname{arcsec}^{2}$ ) - if present at all. Hence, we assume that the polarimetry of the object is not contaminated by dust around the object.

\section{Modelling of the observed polarization}

We interpret the polarimetric and photometric phase curves of Ixion, Quaoar, and Chiron through extensive numerical simulations of coherent backscattering by Rayleigh scatterers. Note that we avoid making an assumption that the fundamental scatterers responsible for the coherent backscattering contribution would be the single particles in the regolith. With the phenomenological modeling currently including the first multipole contribution of an electric dipole, we allow for the possibility that the fundamental scatterers can be the volume and surface inhomogeneities within and on the particles, respectively. Shadowing among the regolith particles is known to contribute to the opposition effect but not to the negative polarization surge. Here, as in Boehnhardt et al. (2004), the shadowing effect manifests itself in the residual slope parameter resulting from the combined coherent backscattering modeling on the polarimetry and photometry.

The coherent-backscattering mechanism is a multiplescattering mechanism for scattering orders higher than the first one. For a recent review of the coherent-backscattering mechanism and its relevance in asteroid studies, see Muinonen et al. (2002) and Muinonen (2004). Note that the computational technique for coherent backscattering accounts for all orders of scattering contributing in a non-negligible way to the backscattering peaks and polarization surges.

In the following we limit ourselves to illustrate the mechanism at the second order of scattering. Let us consider a semiinfinite random medium of discrete scatterers, constrained by a plane-parallel boundary with free space, and an electromagnetic plane wave incident on the random medium from the free space. Let us assume that the incident wave interacts with one of the scatterers, giving rise to first-order scattering, and that, subsequently, the first-order scattered field interacts with another scatterer, giving rise to second-order scattering. Let us assume that the field scattered by the second scatterer escapes the medium and is detected by the observer in the free space. Now there is the reciprocal sequence of scatterings in the opposite direction involving the very same two scatterers. In the exact backward scattering direction, due to the identical lengths of the propagation paths, the two reciprocal wave components interfere constructively whereas, in other directions, the interference characteristics vary. After configurational averaging, a backscattering peak results in the proximity of the backward scattering direction. Focusing in on the polarization characteristics, the interference is selective and tends to invert the linear polarization characteristics in single scattering: for positively polarizing single scattering, coherent backscattering tends to result in negative polarization. The illustration can be readily generalized to higher orders of scattering. In the modeling that follows, all relevant orders of scattering are taken into account.

For the modelling of Ixion, Quaoar, and Chiron, we carried out coherent backscattering computations for a total of 360 spherical media of Rayleigh scatterers, as follows: we assumed 18 different single-scattering albedos

$\tilde{\omega}=0.05,0.10, \ldots, 0.90$ 
and 20 different dimensionless mean free paths

$$
\begin{aligned}
k \ell=2 \pi \ell / \lambda= & 10,20,30, \ldots, 100,120,140, \ldots, \\
& 200,250,300, \ldots, 400,500
\end{aligned}
$$

where $k$ and $\lambda$ are the wave number and wavelength, respectively.

As to the $R$-band geometric albedos $p_{R}$, we adopted $p_{R}=$ 0.23 for Ixion (Stansberry, priv. comm.), 0.11 for Quaoar (calculated using the diameter from Brown \& Trujillo 2004 and our determination of the absolute magnitude), and 0.17 for Chiron (Barucci et al. 2004). In our previous interpretation of Ixion polarimetric data (Boehnhardt et al. 2004), we had assumed $p_{R}=0.1$.

We compared the polarimetric observations against the spherical media composed of monodisperse Rayleigh scatterers with given single-scattering albedo and mean free path (two parameters). The fits were poor: for a fixed geometric albedo, the monodisperse Rayleigh-scattering model tends to result in polarizations that are substantially pronounced as compared to the polarizations observed.

As in Boehnhardt et al. (2004), we then studied a twocomponent Rayleigh-scattering model consisting of dark and bright scatterers. There are five parameters in such a model: two single-scattering albedos and two mean free paths, and the weight factor for the dark component (one minus the weight factor of the bright component). Fixing the geometric albedo fixes the weight factor, reducing the number of free parameters to four. After a systematic study of physically realistic combinations of the two kinds of scatterers, satisfactory fits were obtained for the polarizations of all three objects. For Ixion, the data point at phase angle $0.43^{\circ}$ was omitted as an outlier. The model parameters and rms values of the fits are summarized in Table 5 and the actual fits are depicted in Figs. 2-4. After the polarization fits, approximate brightness fits were obtained by varying the absolute magnitude $H_{R}$ and slope parameter $k_{R}$ of a linear phase dependence multiplying the coherent backscattering contribution (see Figs. 5-7). For Chiron, the observation at $1.8^{\circ}$ phase angle was omitted as outlier.

For all three objects, the dark component shows a mean free path substantially longer than the wavelength. For Chiron, the mean free path of the bright component is also considerably longer than the wavelength. The dark components of Ixion and Quaoar resemble one another and the bright component of Chiron: this is concluded from the similarity of the parameters of the corresponding scatterers on the surfaces of the three objects. The phase curves of Ixion and Chiron resemble each other to an extent where their combined polarimetric and photometric data could be explained using a single two-component scattering model.

The polarimetric observations suggest that Ixion and Chiron could have similar surface structure. It is notable that the geometric albedo ranges of Ixion and Chiron overlap, whereas Quaoar stands out as a darker object. More observational and theoretical work is required for further conclusions.
Table 5. The best fit coherent-backscattering model parameters for Ixion, Quaoar, and Chiron. We give the single-scattering albedos $\tilde{\omega}$ and dimensionless mean free paths $k \ell$ for the dark (subscript d) and bright components (b), the weight of the dark component $w_{\mathrm{d}}$, the rms values of the polarimetric fits, as well as the $R$-band absolute magnitudes $H_{R}$ and slope parameters $k_{R}$.

\begin{tabular}{llll}
\hline \hline & Ixion & Quaoar & Chiron \\
\hline$\tilde{\omega}_{\mathrm{d}}$ & 0.45 & 0.35 & 0.15 \\
$\ell_{\mathrm{d}}$ & 250 & 300 & 120 \\
$\tilde{\omega}_{\mathrm{b}}$ & 0.80 & 0.50 & 0.60 \\
$\ell_{\mathrm{b}}$ & 20 & 10 & 500 \\
$\omega_{\mathrm{d}}$ & 0.74 & 0.46 & 0.14 \\
$\mathrm{rms}$ & $0.029 \%$ & $0.069 \%$ & $0.067 \%$ \\
$H_{R}$ & 3.25 & 2.15 & 5.41 \\
$k_{R}$ & $0.12 \mathrm{deg}^{-1}$ & $0.11 \mathrm{deg}^{-1}$ & $0.041 \mathrm{deg}^{-1}$ \\
\hline
\end{tabular}

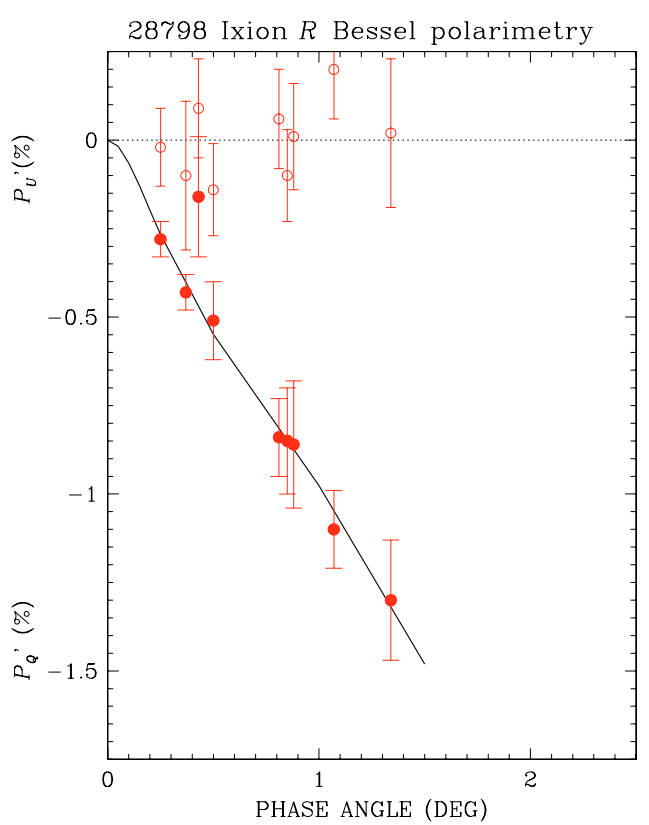

Fig. 2. The observed broadband polarization of 28978 Ixion. The solid symbols refer to $P_{Q}^{\prime}$, i.e., the flux perpendicular to the plane SunObject-Earth minus the flux parallel to that plane, divided by the sum of the two fluxes. The empty circles refer to $P_{U}^{\prime}$ (measured in the scattering plane as explained in the text). Solid line represents the modelling fit to $P_{Q}^{\prime}$. The dotted line shows the expected $P_{U}^{\prime}$ null values.

\section{Discussion and conclusions}

We have presented the results of the first polarimetric observations for a Centaur and a classical disk object in the Kuiper Belt. Together with polarimetric observations of Ixion (Boehnhardt et al. 2004), a representative of the Plutino population in the belt, they give us a first idea about the behavior of linear polarization and intensity of the light reflected by surfaces of Kuiper belt objects and Centaurs. Two Kuiper Belt objects, Ixion and Quaoar, were observed practically in the same range of phase angles $\left(0.25^{\circ}-1.3^{\circ}\right)$. They have shown completely different polarization-phase behaviour. For Ixion the negative polarization rapidly increases with phase angle 


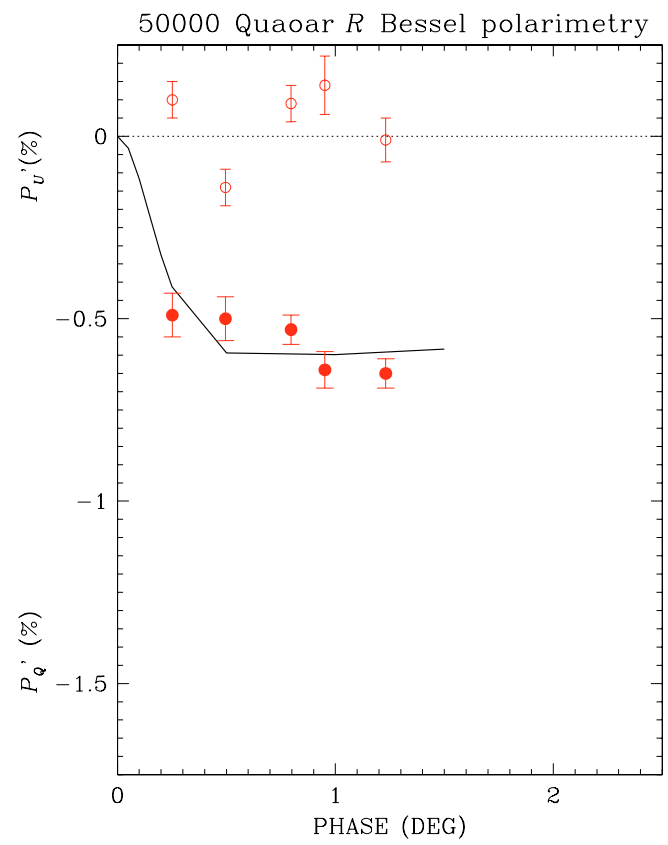

Fig. 3. The broadband polarization of 50000 Quaoar observed in the Bessel $R$ filter. As in Fig. 2, solid symbols refer to $P_{Q}^{\prime}$, and open symbols refer to $P_{U}^{\prime}$. Solid line represents the modelling fit to $P_{Q}^{\prime}$. Dotted lines show the expected null $P_{U}^{\prime}$ values.

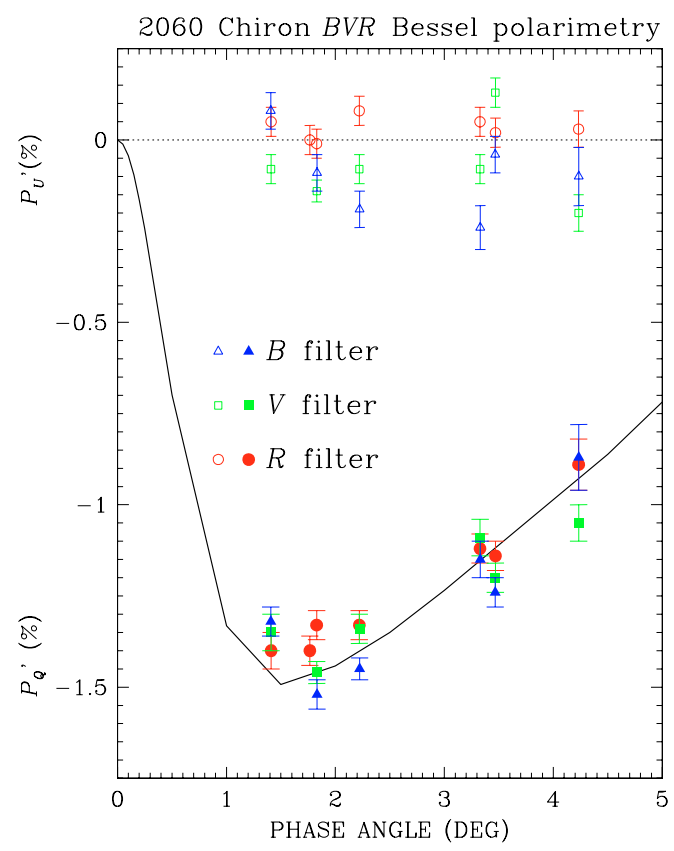

Fig. 4. The observed broadband polarization of 2060 Chiron. As in Fig. 2, the solid symbols refer to $P_{Q}^{\prime}$, and the empty symbols refer to $P_{U}^{\prime}$. Triangles, squares, and circles refer to the polarization measured in the Bessel $B, V$, and $R$ filters, respectively. Solid line represents the modelling fit to $P_{Q}^{\prime}$ for the $R$ filter. The dotted line shows the expected $P_{U}^{\prime}$ null values.

$(-1.02 \pm 0.025 \% / \mathrm{deg})$, while for Quaoar the polarization degree is changed very slowly $(-0.17 \pm 0.10 \% / \mathrm{deg})$. Our polarimetric observations of Chiron were made for larger phase

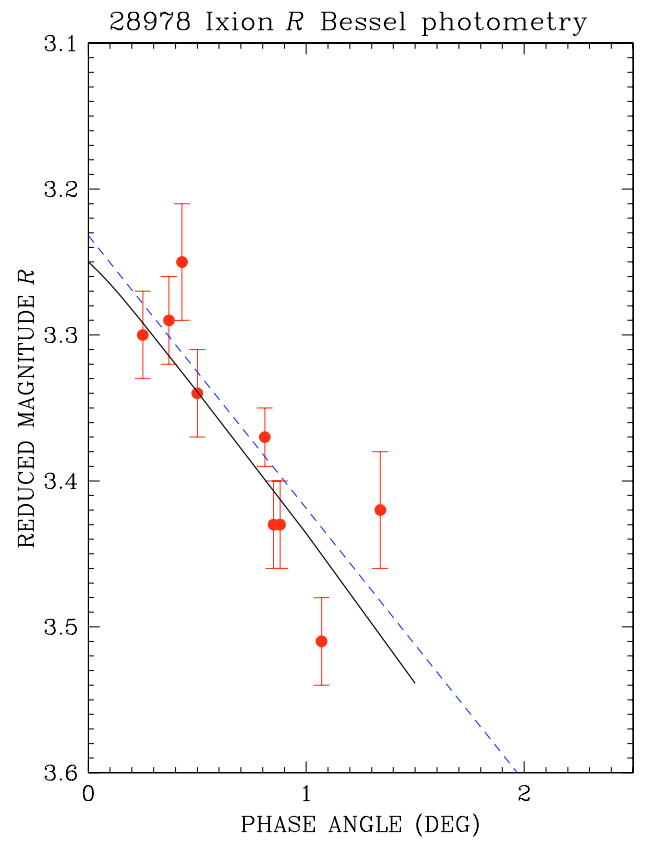

Fig. 5. The observed reduced magnitude $R$ of Ixion. The dashed line represents the best fit obtained with a straight line. The solid line represents the model fit. The scattering around the fits is fully within the amplitude of intrinsic variability of the object.

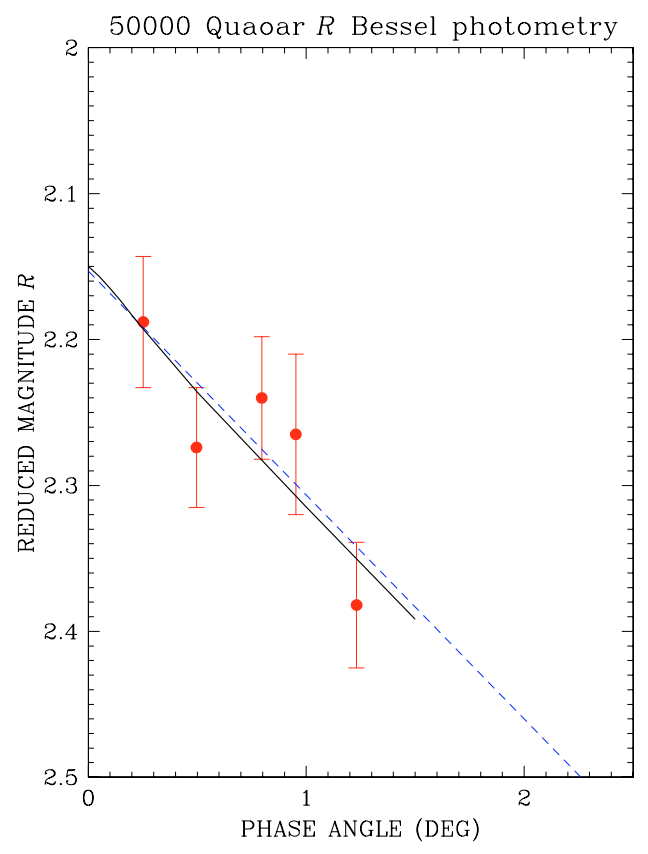

Fig. 6. The observed reduced magnitude $R$ of Quaoar. Symbols are the same as Fig. 5.

angles, $1.4^{\circ}-4.2^{\circ}$. They are characterized by a pronounced branch of negative polarization with a minimum of $1.4-1.5 \%$ at phase angles of $1.5^{\circ}-2.0^{\circ}$. Such polarimetric characteristics are unique among Solar System bodies. For the majority of Solar System bodies the polarization at the phase angle $1^{\circ}$ is characterized by values within $0.1-0.5 \%$. The largest values of the 


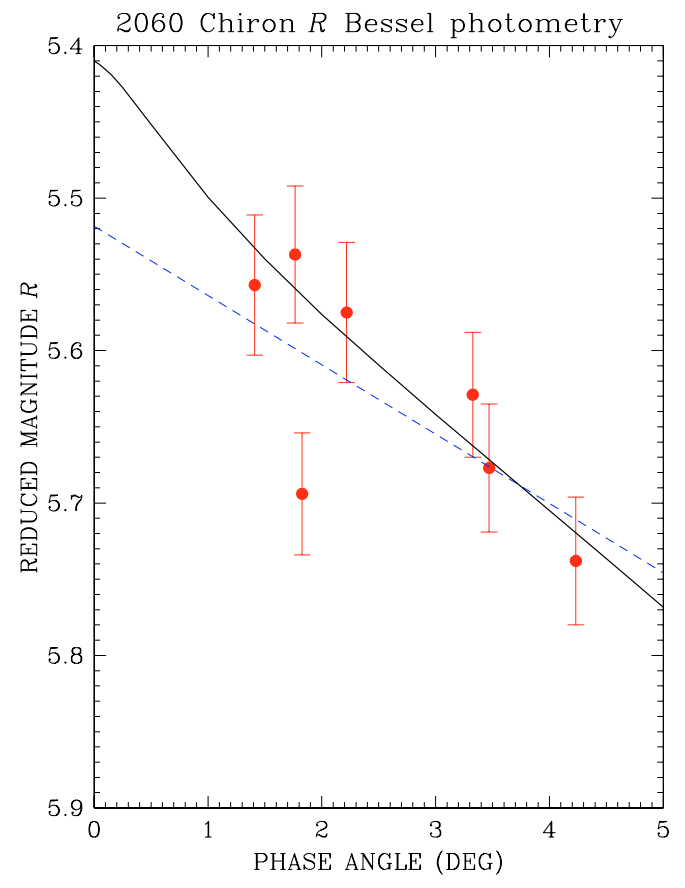

Fig. 7. The observed reduced magnitude $R$ of Chiron. Symbols are the same as Fig. 5.

polarization at $1^{\circ}$ measured for non-TNO objects were $0.83 \%$ for the dark side of Iapetus (albedo $=0.05$ ) and $0.73 \%$ for Saturn Ring A (albedo=0.75) (Rosenbush et al. 2002) that is noticeably smaller than the values obtained at our observations of Ixion and Chiron. Polarimetric observations of Chiron at smaller phase angles are urgently needed for a better modelling, and to compare Chiron data with the polarimetric curve of Ixion and Quaoar. Further observations would be also desirable to identify the inversion angle.

Our modelling has shown that the possible way to explain observed polarization properties of KBOs and Centaur is to assume two-component surface media consisting of dark and bright scatterers. Such a model succeeds in fitting all observed polarimetric and photometric characteristics of the three objects and the two-component modelling is realistic. A more thorough theoretical study is beyond the scope of the present article and should be carried out in the nearest future.

Acknowledgements. The authors wish to thank D. Rabinowitz for sharing his unpublished photometric data of Quaoar which have helped us to verify the calibration of our data, E. Landi Degl'Innocenti and M. Landolfi for their help to write Sect. 3, and O. Hainaut for his help to identify adequate observing periods for our targets (i.e., with no or little risk of background star confusion).

\section{References}

Appenzeller, I. 1967, PASP, 79, 136

Bagnulo, S., Landstreet, J. D., Mason, E., et al. 2006, A\&A, in press [arXiv: astro-ph/0601516]
Barucci, M. A., Cruikshank, D. P., Mottola, S., \& Lazzarin, M. 2003, in Asteroids III, ed. W. F. Jr., Bottke, A. Cellino, P. Paolicchi, \& R. P. Binzel (Tucson: University of Arizona Press), 273

Barucci, A., Doressoundiram, A., \& Cruikshank, D. P. 2004, in Comets II, ed. M. C. Festou, H. U. Keller, \& H. A. Weaver (Tucson: University of Arizona Press), 745, 647

Belskaya, I. N., Shkuratov, Yu. G., Efimov, Yu., et al. 2005, Icarus, 178,213

Boehnhardt, H., Bagnulo, S., Muinonen, K., et al. 2004, A\&A, 415, L21

Brown, M. E., \& Trujillo, C. A. 2004, AJ, 127, 2413

Bus, S. J., A'Hearn, M. F., Schleicher, D. G., \& Bowell, E. 1991, Science, 251, 774

Davies, J. K., McBride, N., Ellison, S. L., Green, S. F., \& Ballantyne, D. R. 1998, Icarus, 134, 213

Davis, L., Specifications for the Aperture Photometry Package 1987, available at http://iraf.noao.edu/docs/photom.html

Duffard, R., Lazzaro, D., Pinto, S., et al. 2002, Icarus, 160, 44

Fornasier, S., Doressoundiram, A., Tozzi, G. P., et al. 2004, A\&A, 421, 353

Foster, M. J., Green, S. F., McBride, N., et al. 1999, Icarus, 141, 408

Gomes, R. 2003, EMP, 92, 29

Groussin, O., Lamy, P., \& Jorda, L. 2004, A\&A, 413, 1163

Jewitt, D. C., \& Luu, J. 2004, Nature, 432, 731

Kelsey, J. D., \& Fix, L. A. 1973, AJ, 184, 633

Landi Degl'Innocenti, E., \& Landolfi, M. 2004, Polarization in Spectral Lines, Astrophysics and Space Library, 307 (Dordrecht: Kluwer Academic Publishers)

Licandro, J., Ghinassi, F., \& Testi, L. 2002, A\&A, 288, L9

Luu, J., \& Jewitt, D. C., 1990, AJ, 100, 913

Luu, J. X., Jewitt, D. C., \& Trujillo, C. 2000, ApJ, 531, L151

Lyot, B. 1929, Ann. Obs. Paris, 8, 1

Marchi, S., Lazzarin, M., Magrin, S., \& Barbieri, C. 2003, A\&A, 408, L17

Meech, K. J., \& Belton, M. J. S. 1990, AJ, 100, 1323

Muinonen, K. 1990, Ph.D. Thesis, University of Helsinki

Muinonen, K., Piironen, J., Shkuratov, Yu. G., Ovcharenko, A., \& Clark, B. E. 2002, in Asteroids III, ed. W. Bottke, R. P. Binzel, A. Cellino, \& P. Paolicchi (Tucson, Arizona, USA: University of Arizona Press), 123

Muinonen, K. 2004, Waves Random Media, 14(3), 365

Ortiz, J. L., Gutierrez, P. J., Sota, A., et al. 2003, A\&A, 409, 130

Patat, F., \& Romaniello, M. 2006, PASP, 118, 146

Pinilla-Alonso, N., Licandro, J., \& Campins, H. 2004, DPS, 36, 1107

Romon-Martin, J., Delahodde, C., Barucci, A., de Bergh, C., \& Peixinho, N. 2003, A\&A, 400, 369

Rosenbush, V., Kiselev, N., Avramchuk, V., \& Mishchenko, M. 2002, in Optics of Cosmic Dust, ed. G. Videen, \& M. Kocifaj, NATO Science Series (Dordrecht, Boston, London: Kluwer Academic Publishers), 191

Rosenbush, V. K., Kiselev, N. N., Shevchenko, V. G., et al. 2005, Icarus, 178, 222

Shkuratov, Yu. G. 1989, Astron. Vestnik, 23, 176

Shkuratov, Yu., Ovcharenko, A., Zubko, E., et al. 2002, Icarus, 159, 396

Shurcliff, W. A. 1962, Polarized light (Cambridge: Harward University Press)

Stansberry, J. A., Cruikshank, D. P., Grundy, W. G., et al. 2005, DPS meeting, No. 37, 5205

Tegler, S. C., Romanishin, W., \& Consolmagno, G. 2003, ApJ, 599, L49 